\title{
Condition assessment of damaged elbow in subsea pipelines
}

\author{
Geon Ho Lee ${ }^{1}$, Jung Kwan Seo, ,* Jeom Kee Paik ${ }^{1,2,3}$ \\ ${ }^{1}$ Department Narval Archtecture and Ocean Enigeering, Pusan National University, Busan, Korea \\ 2 The Korea Ship and Offshore Research Institute (The Lloyd's Register Foundation Research \\ Centre of Excellence), Pusan National University, Busan, Korea \\ ${ }^{3}$ Department of Mechanical Engineering, University College London, UK \\ ${ }^{*}$ Corresponding author. Tel.: +82 51 510-2415. Email address: seojk@pusan.ac.kr (J.K. Seo).
}

\begin{abstract}
The evaluation of the performance of aged structures is essential in the oil and gas industry, where inaccurate predictions of structural performance may lead to significant hazardous consequences. Elbows are critical structures subject to continuous corrosion that can lead to a burst or collapse. It is important to be able to predict both burst strength in continually corroding structures and the behaviour of the structures after critical corrosion takes place. Structural failures due to a significant reduction in wall thickness make it very complicated for pipeline operators to maintain pipeline serviceability. This paper discusses the plastic limit pressure of elbows without defects and with several local thinned areas. Finite element analysis (FEA) and the Goodall formula were used to evaluate the new formula. The results of the FEA show that the limit load of elbows under internal pressure differs with position and depth of damage. An empirical formula for the limit load of elbows with local thinned areas is proposed. Eight sizes of elbow were considered in this study, with $\mathrm{Rm} / \mathrm{t}$ of 5, 6, 7.5, 9, 12, 15, 20 and 25. This range covers most of the piping used in high-pressure environments, such as nuclear and subsea situations. The method of numerical analysis was validated by experiment and with FEA results from the literature. The results of the study can be applied to both the operation and assessment of pipelines. A formula to predict the maximum burst strength of damaged elbows is presented, which will enable more accurate estimates of the time until pipelines need to be replaced or repaired.
\end{abstract}

Keywords: Elbow, corrosion, burst pressure, 22g steel, local thin area, condition assessment, pipeline serviceability, modified Goodall formula

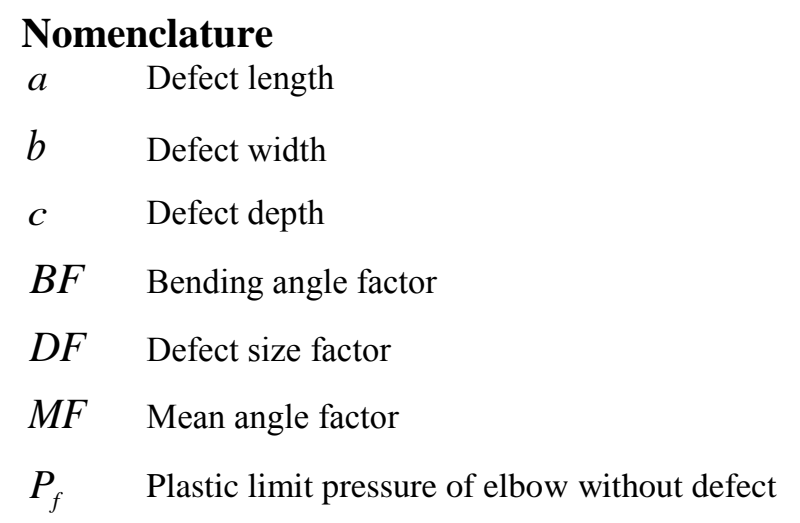


$P_{f d} \quad$ Plastic limit pressure of elbow with defect

$R_{b} \quad$ Elbow bending radius

$R_{m} \quad$ Elbow mean radius

$t \quad$ Elbow wall thickness

TES Twice-elastic-slope

$\alpha \quad$ Circumferential mean angle from the crown of the elbow $\left(+90^{\circ} \sim-90^{\circ}\right)$

$\beta \quad$ Circumferential bending angle from the crown of the elbow $\left(0^{\circ} \sim 45^{\circ}\right)$

$\sigma_{f} \quad$ Flow stress (Goodall Formula)

$\sigma_{H(\max )}$ Maximum stress distribution of elbow

$\sigma_{H(\min )}$ Minimum stress distribution of elbow

$\sigma_{u} \quad$ Material ultimate tensile stress

$\sigma_{y} \quad$ Material yield tensile stress

\section{Introduction}

One of the critical problems in the gas pipeline industry is corrosion. Elbows are considered to be critical components in a piping system, and are also the structures in gas pipelines that are most easily damaged by corrosion. The maintenance of gradually corroding metal elbows is an especially important issue for the pipeline industry.

Piping systems are subjected to loads such as internal pressure, moment, torsion, and dead weight, and as maximum stress occurs in the elbows these parts of a pipeline fail earlier than a straight pipe (Guo, 1999). Defects caused by corrosion, erosion, mechanical damage or crack polishing also reduce the structural integrity of pipelines and may affect the safe operation of the system.

Calculation of the limit loads of elbows with defects is essential to evaluate the safe operation of structures (Han and Liu, 1998). The limit load acceptance criteria of a straight pipe with defects cannot be used to assess an elbow with identical defects due to elbows' characteristic stress intensity (Zhang et al., 2001). Results have been obtained through experimental evaluation of the effect of local wall thinning on the failure pressure of several elbows (Kim et al., 2009), and a series of burst tests using real-scale elbows were performed to develop a numerical formula.

The formulas were extracted via experimental data on one size of elbow with local wall thinning in the centre of the bending angle. The effects of local wall thinning on the plastic limit loads of elbows using geometrically linear finite element limit analyses (Kim et al., 2008) were derived to provide closed-form plastic limit load solutions for elbows under in-plane bending and internal pressure via three-dimensional (3D) geometrically linear FE limit analyses using elastic-perfectly plastic materials. FEA of elastic stresses for $90^{\circ}$ elbows under in-plane bending (An et al., 2011) shows that for $90^{\circ}$ elbows, an in-plane bending moment produces not only an axial membrane stress component but also axial and hoop bending stress components. Furthermore, the magnitudes of these stress components depend strongly on the mean radius- 
to-thickness ratio, the circumferential location and the longitudinal location. Maximum stresses tend to occur in the centre of the elbow at or near the crown. Engineers' methods to determine limit load values include the twice-elastic-slope, three times-elastic-slope, twice-elastic defect, tangent intersection, zerocurvature and $0.2 \%$ residual strain method.

The limit load determination of this study is based on load-strain curves using the TES method. An investigation into the effect of internal pressure on the in-plane collapse moment of elbows (Chattopadhyay, 2002) was carried out to evaluate the collapse moment of several sizes of elbow and to validate the limit load of elbows obtained by the TES method from the moment versus end rotation curve. Quantification of the yield strength to elastic modulus ratio effect on TES plastic loads from FE limit analyses of elbows (Lee. et al., 2009) was considered to qualify the effect of the yield strength to elastic modulus ratio on TES loads on $90^{\circ}$ elbow bending loads.

A comparison of existing plastic collapse load solutions with experimental data for $90^{\circ}$ elbows (Han et al., 2012) was performed to compare with existing methods of deriving collapse loads for $90^{\circ}$ elbows. The limit load value was also determined for a seismic fragility analysis of a seismically isolated nuclear power plant's piping system (Firoozabad et al., 2015) using the TES method to identify the critical points in the system. The simulation results were validated with a monotonic and cyclic test of the critical points, and the conditional mean spectrum method was used to scale the selected records. The fragility curves of the NPP piping system were estimated and the computation of the fragility parameters was addressed. Elastic, shakedown and plastic limit loads for $90^{\circ}$ elbows under constant internal pressure and cyclic in-plane bending were presented via FEA (Oh et al., 2008).

The effects of the elbow geometry and of the large geometry change were systematically investigated. By normalising the in-plane bending moment by the plastic limit load solution of Calladine (Calladine, 1974), the shakedown diagram was found to be close to unity up to a certain value of normalised pressure and then to decrease almost linearly with increasing normalised pressure. Several sizes of elbow with several positions of defect were also calculated by FE analysis, and the influence of the defect dimensions on limit load was determined (Wang et al., 2005). The plastic limit pressure of elbows without defects and with a local thinned area in the extrados as derived from FEM analysis and experimental data was compared with the Goodall formula. An empirical formula for the limit load of elbows with local thinned areas in the extrados has been proposed by data fitting FEA results that were validated by experiment (Duan and Shen, 2006). However, the position of the defects was not considered, and the research was also limited to defects in the centre of the extrados.

In the same study, an elastic-perfectly plastic material model was considered. The results prompted a consideration of the plastic limit load of elbows with defects all around the bending angle, both internally and externally. A stress-strain curve of material was adopted to prevent conservative results. An evaluation of the plastic limit load of elbows without defects for all models was performed to validate the numerical analysis method. 


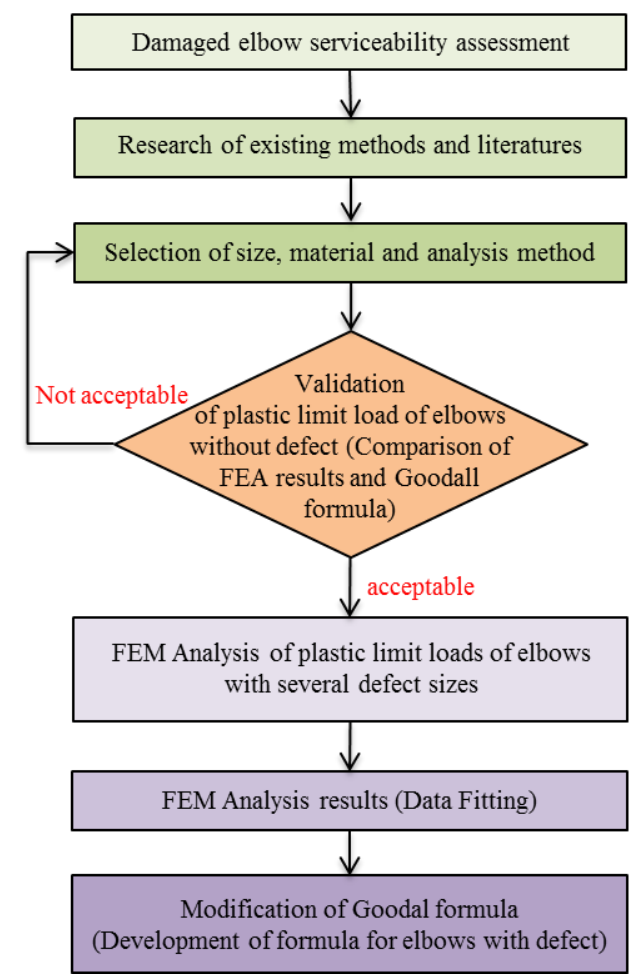

Fig. 1. Overall procedure for the evaluation of the maximum limit load of steel elbow with defects under internal pressure

In this study, the burst strength capacity of an elbow with a defect was evaluated using empirical models and a numerical analysis of internal pressure. The analysis results were then assessed and compared using a numerical analysis (ANSYS, 2012) and empirical formula (Goodall, 1978) to develop a new method for the evaluation of the maximum plastic limit load of elbows with defects.

\section{Finite Element Analysis}

\subsection{Elbow size and modelling}

Eight sizes of elbow with a $90^{\circ}$ bending angle $\left(\mathrm{R}_{\mathrm{b}}\right.$ and $\mathrm{R}_{\mathrm{m}}$ are Elbow bending radius and mean radius, respectively) were considered in this study. For all sizes $R_{b} / R_{m}=3$ and $R_{m} / t$ was $5,6,7.5,9,12,15,20$ and 25. These choices reflect the sizes of pipeline most applicable to high-pressure environments, such as in the oil, gas and nuclear industries (Srivastava et al., 2011). Table 1 shows detailed information about each elbow. Straight pipes with a diameter identical to the elbow diameter and a length of $1,000 \mathrm{~mm}$ were added to either end of each elbow.

Table 1. Geometry of the elbows used in the experiment

\begin{tabular}{lccccc}
\hline & $\begin{array}{c}\mathbf{R}_{\mathbf{m}} \\
(\mathbf{m m})\end{array}$ & $\begin{array}{c}\mathbf{R}_{\mathbf{b}} \\
(\mathbf{m m})\end{array}$ & $\begin{array}{c}\mathbf{t} \\
(\mathbf{m m})\end{array}$ & $\mathbf{R}_{\mathbf{b}} / \mathbf{R}_{\mathbf{m}}$ & $\mathbf{R}_{\mathbf{m}} / \mathbf{t}$ \\
\hline Model-01 & 184.73 & 554.19 & 36.95 & 3 & 5.0 \\
\hline Model-02 & 187.57 & 562.71 & 31.26 & 3 & 6.0 \\
\hline Model-03 & 190.50 & 571.50 & 25.40 & 3 & 7.5 \\
\hline Model-04 & 192.51 & 577.53 & 21.39 & 3 & 9.0 \\
\hline
\end{tabular}




\begin{tabular}{llllll} 
Model-05 & 195.07 & 585.21 & 16.26 & 3 & 12.0 \\
\hline Model-06 & 196.65 & 589.95 & 13.11 & 3 & 15.0 \\
\hline Model-07 & 198.24 & 594.72 & 9.91 & 3 & 20.0 \\
\hline Model-08 & 199.22 & 597.66 & 7.97 & 3 & 25.0 \\
\hline
\end{tabular}

Three sizes of defect were assumed for the analysis of the maximum limit loads. Defects were assumed to be rectangular, with a length (a), width (b) and depth (c). The dimensions of the defects are shown in Table 2. The defects were applied to the models externally and internally.

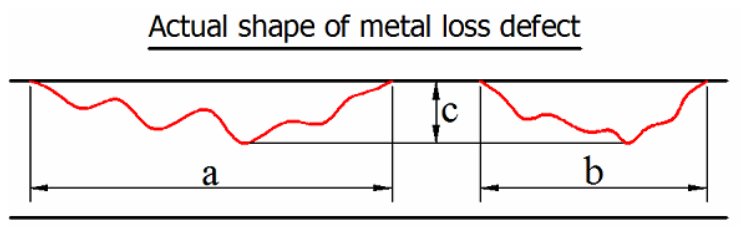

Rectangular assumption shape of metal loss defect

Fig. 2. Assumed shape of the defects

The shapes of the defects are shown in Fig. 2. The actual defects were simplified to a rectangular model to make the FEA modelling possible.

Table 2. Geometry of the defects

\begin{tabular}{lccc}
\hline & $\mathbf{a}$ & $\mathbf{b}$ & $\mathbf{c}$ \\
$(\mathbf{m m})$ & $(\mathbf{m m})$ & $(\mathbf{m m})$ \\
\hline Defect-1 & 150 & 75 & $0.25 \mathrm{t}$ \\
\hline Defect-2 & 200 & 100 & $0.50 \mathrm{t}$ \\
\hline Defect-3 & 300 & 150 & $0.75 \mathrm{t}$ \\
\hline
\end{tabular}

\subsection{Material modelling}

The elbows were $20 \mathrm{~g}$ steel, which is applicable in high-pressure environments such as those of the oil, gas and nuclear industries. The real stress-strain curve of this material was found by experiment with an MTS testing machine (Duan and Shen, 2006). The real stress-strain curves for the steel in the axial and circumferential directions of the pipe are different. In this case is assumed that the stress-strain curves for both directions are same. These material properties were used for the FEA and the calculation of the Goodall formula.

Table 3. Material properties of 20g steel found by experiment (Duan and Shen, 2006).

\begin{tabular}{cccc}
\hline $\begin{array}{c}\text { Young's Modulus } \\
(\mathbf{M P a})\end{array}$ & $\begin{array}{c}\text { Poisson's } \\
\text { Ratio }\end{array}$ & $\begin{array}{c}\text { Yield Strength } \\
(\mathbf{M P a})\end{array}$ & $\begin{array}{c}\text { Ultimate Strength } \\
(\mathbf{M P a})\end{array}$ \\
\hline $1.7397 \times 10^{5}$ & 0.3 & 345 & 517 \\
\hline
\end{tabular}

The kinematic hardening multi-linear material model was adopted for the FEA. There are six segments in plastic stages and one segment in an elastic stage as shown in Fig. 3. 


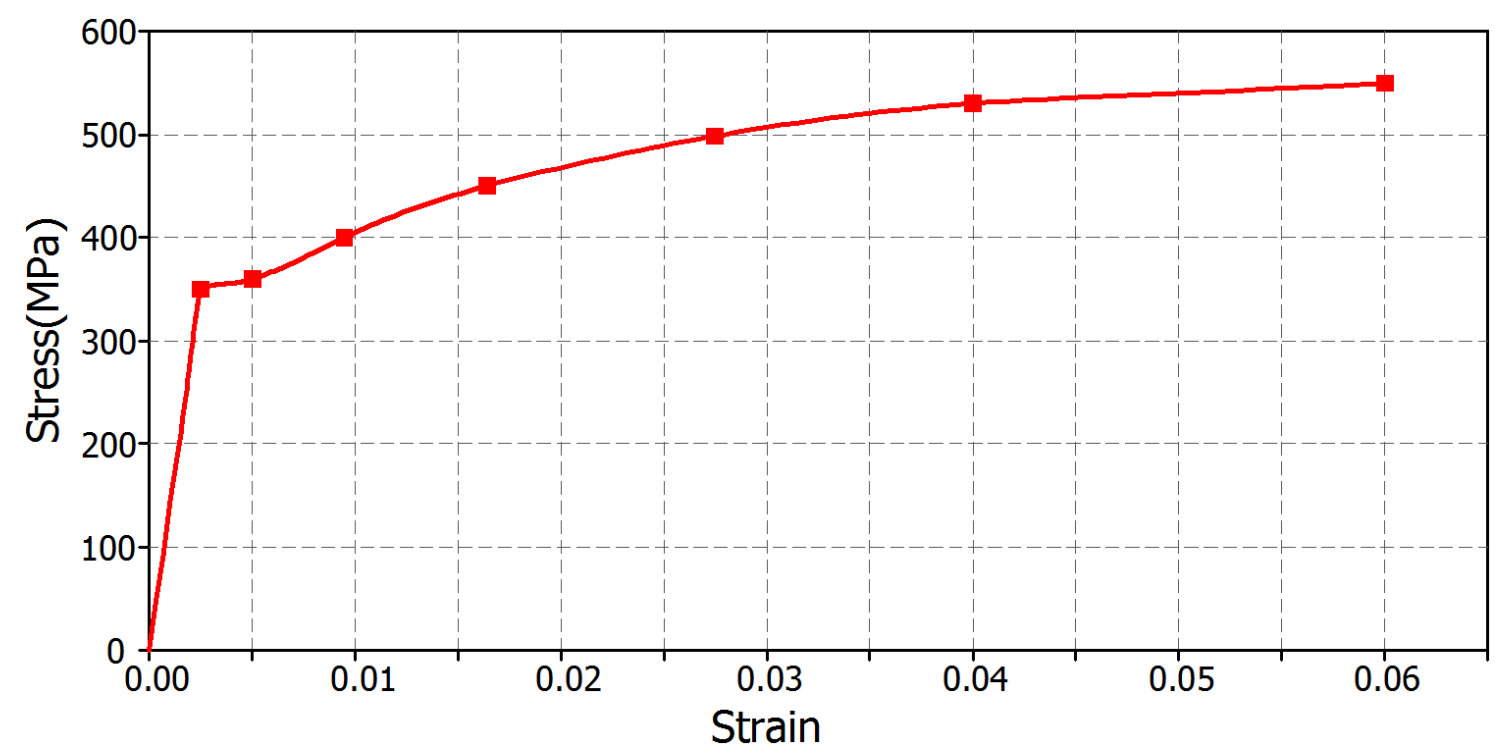

Fig.3. Real stress-strain curve of the $20 \mathrm{~g}$ steel

\subsection{Load and boundary condition}

Both ends of the elbows were fixed in the $\mathrm{X}, \mathrm{Y}$ and $\mathrm{Z}$ directions to restrict their movement. An internal pressure $\mathrm{P}$ was applied to all internal surfaces. The boundary condition and load condition of the elbows in the FEA is shown in Fig.4.

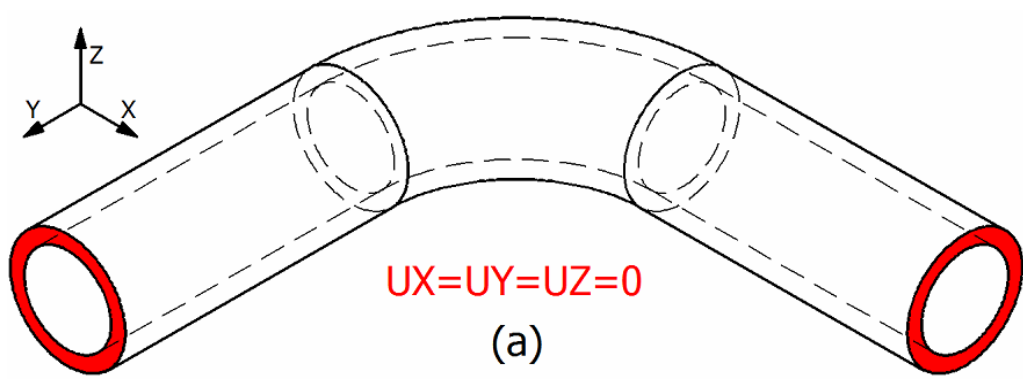

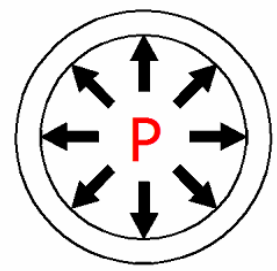

(b)

Fig.4. (a) Applied boundary condition. (b) Applied load

\subsection{Meshing}

The structure was first modelled by a 3D CAD modeller and exported to ANSYS for the numerical simulation. A full scale 8-node isoparametric brick (Solid 185) with a reduced integration option model was chosen for the target structure (elbow). An inelastic multi-linear material model was also used to simulate the structures. A set of mesh convergence tests for several cases were performed to determine the appropriate size and quantity of mesh. It is desirable to find the minimum number of elements that give a converged solution. A total of 5,080 elements for the elbow model without defects, and 5,072 elements and 6,533 nodes for the elbow model with defects were symetrically designed. The FE modelling of the elbows is shown in Fig. 5. 


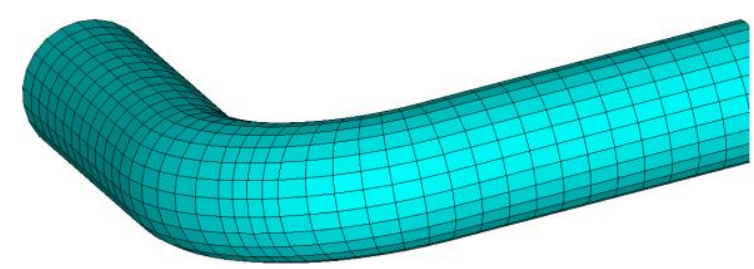

(a)

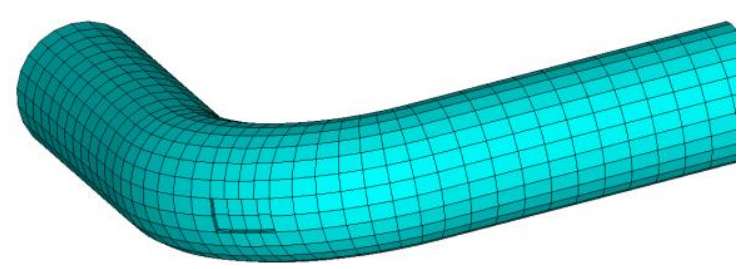

(b)

Fig.5. (a) Finite element meshing of elbow without defect. (b) Finite element meshing of elbow with defect.

\subsection{Limit load}

The definition of the ideal limit load when the load corresponds to the limit state is where the load stops increasing but the strain rate of displacement continues increasing to infinity. The material is assumed to be elastic-perfectly plastic with only small displacements. Such ideal material in reality does not exist because of strain-hardening and geometry hardening or weakening. Therefore, as mentioned above, engineers propose several methods of determining the limit load. The limit load determination in the present study was based on the load-strain curves using the twice-elastic-slope (TES) method. The ASME Boilers and Pressure Vessel Code (ASME BPVC, 2010) describes the TES method in detail. The strain in the loadstrain curve is the maximum von-Mises strain of the elbow. Fig. 6 shows the times-elastic-slope method indicated in ASME BPVC 2010.

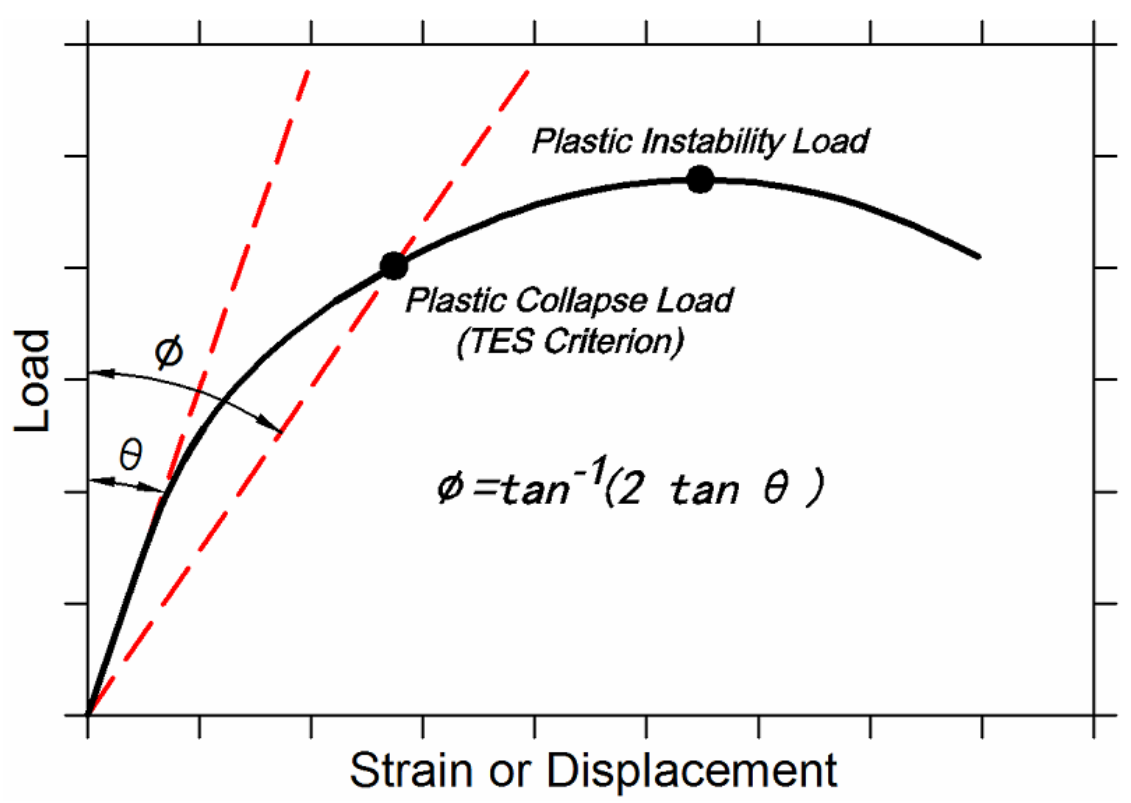

Fig.6. Times-Elastic-Slope method (TES criterion)

There are many examples in the literature of using the TES method to restrict the maximum allowable load of elbows (Firoozabad et al., 2015).

\subsection{Analysis of the maximum limit loads of elbows without defect}


The maximum limit loads of elbows without defect are shown in Table 4. An FEA was implemented for all elbow models without defects. The results were compared with the Goodall formula (1). Goodall proposed the following formula for the limit pressure $P_{f}$ for an elbow without any damage:

$$
P_{f}=\frac{\sigma_{f} t}{R_{m}} \frac{1-R_{m} / R_{b}}{1-R_{m} /\left(2 R_{b}\right)}
$$

The flow stress in the formula is defined by $\sigma_{f}=\left(\sigma_{y}+\sigma_{u}\right) / 2$. The FEA method used is identical to the method for analysis of elbows without defect suggested by Duan and Shen (2006). Comparison of the FEA and the Goodall formula confirms that the FE method of assessing the limit load of elbows is correct and comparable with the formula. The errors were less than $2 \%$ for all models.

Table 4. Comparison of the FEA and Goodall formula results

\begin{tabular}{|c|c|c|c|c|c|c|}
\hline & \multirow{2}{*}{$\underset{(\mathbf{m m})}{\mathbf{R m}}$} & \multirow{2}{*}{$\begin{array}{c}\mathbf{R b} \\
(\mathbf{m m})\end{array}$} & \multirow{2}{*}{$\begin{array}{c}\mathbf{t} \\
(\mathbf{m m})\end{array}$} & \multicolumn{2}{|c|}{ Limit Loads (MPa) } & \multirow{2}{*}{$\frac{\text { Errors }(\%)}{(\text { Plg-Plf }) /\left(\mathbf{P}_{L F}\right)}$} \\
\hline & & & & FEA (PLF) & Goodall (PLG) & \\
\hline Model-01 & 184.73 & 554.19 & 36.95 & 69.48 & 69.13 & -0.51 \\
\hline Model-02 & 187.57 & 562.71 & 31.26 & 58.16 & 57.48 & -0.55 \\
\hline Model-03 & 190.50 & 571.50 & 25.40 & 46.55 & 46.21 & -0.73 \\
\hline Model-04 & 192.51 & 577.53 & 21.39 & 38.41 & 38.39 & -0.04 \\
\hline Model-05 & 195.07 & 585.21 & 16.26 & 28.73 & 38.82 & 0.25 \\
\hline Model-06 & 196.65 & 589.95 & 13.11 & 22.98 & 23.03 & 0.22 \\
\hline Model-07 & 198.24 & 594.72 & 9.91 & 17.23 & 17.38 & 0.31 \\
\hline Model-08 & 199.22 & 597.66 & 7.97 & 13.76 & 13.93 & 0.47 \\
\hline
\end{tabular}

\subsection{Analysis of the maximum limit load of elbows with defect}

A numerical FEA for all elbow models of a different size and defect position was performed to determine the maximum limit load. The modelling, meshing, load condition, boundary condition and material properties of elbows with defect were identical to elbows without defect.

Table 5. FEA results for elbows with three sizes of defect, three angles of $\beta$ and five angles of $\alpha$

\begin{tabular}{|c|c|c|c|c|c|c|c|c|c|c|c|c|c|c|c|}
\hline \multicolumn{8}{|c|}{ Model-01 } & \multicolumn{8}{|c|}{ Model-o2 } \\
\hline$\alpha$ & $\beta$ & Defect & $\begin{array}{c}\text { FEM } \\
(\mathrm{MPa})\end{array}$ & Defect & $\begin{array}{l}\text { FEM } \\
(\mathrm{MPa})\end{array}$ & Defect & $\begin{array}{l}\text { FEM } \\
(\mathrm{MPa})\end{array}$ & $\alpha$ & $\beta$ & Defect & $\begin{array}{l}\text { FEM } \\
(\mathrm{MPa})\end{array}$ & Defect & $\begin{array}{l}\text { FEM } \\
\text { (MPa) }\end{array}$ & defect & $\begin{array}{c}\text { FEM } \\
(\mathrm{MPa})\end{array}$ \\
\hline \multirow{10}{*}{$0^{\circ}$} & $\left(+90^{\circ}\right)$ & \multirow{5}{*}{$\begin{array}{l}\text { External } \\
\text { Defect-1 }\end{array}$} & $\begin{array}{r}69.48 \\
\end{array}$ & \multirow{5}{*}{$\begin{array}{l}\text { External } \\
\text { Defect-2 }\end{array}$} & 68.60 & \multirow{5}{*}{$\begin{array}{l}\text { External } \\
\text { Defect-3 }\end{array}$} & 40.32 & \multirow{10}{*}{$0^{\circ}$} & $\left(+90^{\circ}\right)$ & \multirow{5}{*}{$\begin{array}{l}\text { External } \\
\text { Defect-1 }\end{array}$} & 58.16 & \multirow{5}{*}{$\begin{array}{l}\text { External } \\
\text { Defect-2 }\end{array}$} & 57.62 & \multirow{5}{*}{$\begin{array}{l}\text { External } \\
\text { Defect-3 }\end{array}$} & 33.79 \\
\hline & $\left(+45^{\circ}\right)$ & & 69.48 & & 67.90 & & 38.98 & & $\left(+45^{\circ}\right)$ & & 58.16 & & 56.76 & & 32.47 \\
\hline & $\left(0^{\circ}\right)$ & & 69.48 & & 61.50 & & 37.15 & & $\left(0^{\circ}\right)$ & & 58.16 & & 51.60 & & 30.96 \\
\hline & $\left(-45^{\circ}\right)$ & & 62.40 & & 56.12 & & 33.92 & & $\left(-45^{\circ}\right)$ & & 52.87 & & 45.37 & & 28.13 \\
\hline & $\left(-90^{\circ}\right)$ & & 60.17 & & 52.48 & & 31.91 & & $\left(-90^{\circ}\right)$ & & 49.32 & & 43.01 & & 26.37 \\
\hline & $\left(+90^{\circ}\right)$ & \multirow{5}{*}{$\begin{array}{l}\text { Internal } \\
\text { Defect-1 }\end{array}$} & 69.48 & \multirow{5}{*}{$\begin{array}{c}\text { Internal } \\
\text { Defect-2 }\end{array}$} & 69.44 & \multirow{5}{*}{$\begin{array}{l}\text { Internal } \\
\text { Defect-3 }\end{array}$} & 40.45 & & $\left(+90^{\circ}\right)$ & \multirow{5}{*}{$\begin{array}{l}\text { Internal } \\
\text { Defect-1 }\end{array}$} & 58.16 & \multirow{5}{*}{$\begin{array}{l}\text { Internal } \\
\text { Defect-2 }\end{array}$} & 57.74 & \multirow{5}{*}{$\begin{array}{l}\text { Internal } \\
\text { Defect-3 }\end{array}$} & 33.61 \\
\hline & $\left(+45^{\circ}\right)$ & & 69.48 & & 68.74 & & 38.77 & & $\left(+45^{\circ}\right)$ & & 58.16 & & 56.71 & & 32.37 \\
\hline & $\left(0^{\circ}\right)$ & & 69.48 & & 60.98 & & 37.35 & & $\left(0^{\circ}\right)$ & & 57.72 & & 51.23 & & 30.62 \\
\hline & $\left(-45^{\circ}\right)$ & & 61.70 & & 55.82 & & 33.74 & & $\left(-45^{\circ}\right)$ & & 52.58 & & 45.13 & & 27.98 \\
\hline & $\left(-90^{\circ}\right)$ & & 59.31 & & 52.20 & & 31.75 & & $\left(-90^{\circ}\right)$ & & 47.59 & & 43.10 & & 26.48 \\
\hline \multirow{10}{*}{$22.5^{\circ}$} & $\left(+90^{\circ}\right)$ & \multirow{5}{*}{$\begin{array}{l}\text { External } \\
\text { Defect-1 }\end{array}$} & 69.48 & \multirow{5}{*}{$\begin{array}{l}\text { External } \\
\text { Defect-2 }\end{array}$} & 69.44 & \multirow{5}{*}{$\begin{array}{l}\text { External } \\
\text { Defect-3 }\end{array}$} & 40.74 & \multirow{10}{*}{$22.5^{\circ}$} & $\left(+90^{\circ}\right)$ & \multirow{5}{*}{$\begin{array}{l}\text { External } \\
\text { Defect-1 }\end{array}$} & 58.16 & \multirow{5}{*}{$\begin{array}{l}\text { External } \\
\text { Defect-2 }\end{array}$} & 57.74 & \multirow{5}{*}{$\begin{array}{l}\text { External } \\
\text { Defect-3 }\end{array}$} & 33.96 \\
\hline & $\left(+45^{\circ}\right)$ & & 69.48 & & 67.90 & & 39.38 & & $\left(+45^{\circ}\right)$ & & 58.16 & & 56.94 & & 32.57 \\
\hline & $\left(0^{\circ}\right)$ & & 69.48 & & 61.97 & & 37.61 & & $\left(0^{\circ}\right)$ & & 58.16 & & 51.76 & & 31.09 \\
\hline & $\left(-45^{\circ}\right)$ & & 63.10 & & 55.82 & & 34.59 & & $\left(-45^{\circ}\right)$ & & 53.43 & & 45.61 & & 28.21 \\
\hline & $\left(-90^{\circ}\right)$ & & 59.85 & & 51.92 & & 32.01 & & $\left(-90^{\circ}\right)$ & & 49.60 & & 43.14 & & 26.48 \\
\hline & $\left(+90^{\circ}\right)$ & \multirow{5}{*}{$\begin{array}{l}\text { Internal } \\
\text { Defect-1 }\end{array}$} & 69.48 & \multirow{5}{*}{$\begin{array}{l}\text { Internal } \\
\text { Defect-2 }\end{array}$} & 69.30 & & 40.66 & & $\left(+90^{\circ}\right)$ & \multirow{5}{*}{$\begin{array}{l}\text { Internal } \\
\text { Defect-1 }\end{array}$} & 58.16 & \multirow{5}{*}{$\begin{array}{l}\text { Internal } \\
\text { Defect-2 }\end{array}$} & 57.33 & \multirow{5}{*}{$\begin{array}{l}\text { Internal } \\
\text { Defect-3 }\end{array}$} & 33.85 \\
\hline & $\left(+45^{\circ}\right)$ & & 69.48 & & 68.11 & & 39.50 & & $\left(+45^{\circ}\right)$ & & 58.16 & & 56.71 & & 32.57 \\
\hline & $\left(0^{\circ}\right)$ & & 69.48 & & 61.63 & Internal & 37.54 & & $\left(0^{\circ}\right)$ & & 57.89 & & 51.23 & & 30.71 \\
\hline & $\left(-45^{\circ}\right)$ & & 63.80 & & 55.53 & & 34.45 & & $\left(-45^{\circ}\right)$ & & 52.87 & & 45.37 & & 28.13 \\
\hline & $\left(-90^{\circ}\right)$ & & 59.85 & & 52.37 & & 32.11 & & $\left(-90^{\circ}\right)$ & & 49.74 & & 43.23 & & 26.43 \\
\hline & $\left(+90^{\circ}\right)$ & External & 69.48 & External & 69.37 & External & 40.95 & 150 & $\left(+90^{\circ}\right)$ & External & 58.16 & External & 57.92 & External & 34.13 \\
\hline $45^{\circ}$ & $\left(+45^{\circ}\right)$ & Defect-1 & 69.48 & Defect-2 & 68.39 & Defect-3 & 39.59 & $45^{\circ}$ & $\left(+45^{\circ}\right)$ & Defect-1 & 58.16 & Defect-2 & 57.05 & Defect-3 & 32.74 \\
\hline
\end{tabular}




\begin{tabular}{|c|c|c|c|c|}
\hline & $\left(0^{\circ}\right)$ & & 69.48 & \\
\hline & $\left(-45^{\circ}\right)$ & & 64.22 & \\
\hline & $\left(-90^{\circ}\right)$ & & 60.82 & \\
\hline & $\left(+90^{\circ}\right)$ & & 69.48 & \\
\hline & $\left(+45^{\circ}\right)$ & & 69.48 & \\
\hline & $\left(0^{\circ}\right)$ & $\begin{array}{l}\text { Internal } \\
\text { Defect-0 }\end{array}$ & 69.48 & $\begin{array}{l}\text { Internal } \\
\text { Defect-2 }\end{array}$ \\
\hline & $\left(-45^{\circ}\right)$ & & 64.15 & \\
\hline & $\left(-90^{\circ}\right)$ & & 60.51 & \\
\hline & & & М o & $\overline{\text { el - o 3 }}$ \\
\hline$\alpha$ & $\beta$ & Defect & $\begin{array}{l}\text { FEM } \\
\text { (MPa) }\end{array}$ & Defect \\
\hline & $\left(+90^{\circ}\right)$ & & 46.55 & \\
\hline & $\left(+45^{\circ}\right)$ & & 46.55 & \\
\hline & $\left(0^{\circ}\right)$ & $\begin{array}{l}\text { External } \\
\text { Defect-1 }\end{array}$ & 46.24 & $\begin{array}{l}\text { External } \\
\text { Dect-2 }\end{array}$ \\
\hline & $\left(-45^{\circ}\right)$ & & 42.23 & \\
\hline $0^{\circ}$ & $\left(-90^{\circ}\right)$ & & 38.50 & \\
\hline $0^{\circ}$ & $\left(+90^{\circ}\right)$ & & 46.55 & \\
\hline & $\left(+45^{\circ}\right)$ & & 46.55 & \\
\hline & $\left(0^{\circ}\right)$ & $\begin{array}{l}\text { Internal } \\
\text { Defect. }\end{array}$ & 46.06 & $\begin{array}{l}\text { Internal } \\
\text { Pect }\end{array}$ \\
\hline & $\left(-45^{\circ}\right)$ & & 42.68 & \\
\hline & $\left(-90^{\circ}\right)$ & & 38.63 & \\
\hline & $\left(+90^{\circ}\right)$ & & 46.55 & \\
\hline & $\left(+45^{\circ}\right)$ & & 46.55 & \\
\hline & $\left(0^{\circ}\right)$ & $\begin{array}{l}\text { External } \\
\text { Defotate }\end{array}$ & 46.34 & External \\
\hline & $\left(-45^{\circ}\right)$ & & 42.88 & \\
\hline $225^{\circ}$ & $\left(-90^{\circ}\right)$ & & 39.07 & \\
\hline $22.5^{\circ}$ & $\left(+90^{\circ}\right)$ & & 46.55 & \\
\hline & $\left(+45^{\circ}\right)$ & & 46.55 & \\
\hline & $\left(0^{\circ}\right)$ & $\begin{array}{l}\text { Internal } \\
\text { Defect-1 }\end{array}$ & 46.29 & $\begin{array}{l}\text { Internal } \\
\text { Defect-2 }\end{array}$ \\
\hline & $\left(-45^{\circ}\right)$ & & 42.91 & \\
\hline & $\left(-90^{\circ}\right)$ & & 39.42 & \\
\hline & $\left(+90^{\circ}\right)$ & & 46.55 & \\
\hline & $\left(+45^{\circ}\right)$ & & 46.55 & \\
\hline & $\left(0^{\circ}\right)$ & $\begin{array}{l}\text { External } \\
\text { Defect-1 }\end{array}$ & 46.43 & $\begin{array}{l}\text { External } \\
\text { Defect-2 }\end{array}$ \\
\hline & $\left(-45^{\circ}\right)$ & & 43.14 & \\
\hline & $\left(-90^{\circ}\right)$ & & 39.73 & \\
\hline $45^{\circ}$ & $\left(+90^{\circ}\right)$ & & 46.55 & \\
\hline & $\left(+45^{\circ}\right)$ & & 46.55 & \\
\hline & $\left(0^{\circ}\right)$ & Internal & 46.31 & Internal \\
\hline & $\left(-45^{\circ}\right)$ & & 43.01 & \\
\hline & $\left(-90^{\circ}\right)$ & & 39.51 & \\
\hline & & & M o & el-05 \\
\hline$\alpha$ & $\beta$ & Defect & $\begin{array}{c}\text { FEM } \\
\text { (MPa) }\end{array}$ & Defect \\
\hline & $\left(+90^{\circ}\right)$ & & 28.74 & \\
\hline & $\left(+45^{\circ}\right)$ & & 28.74 & \\
\hline & $\left(0^{\circ}\right)$ & $\begin{array}{l}\text { External } \\
\text { Defect-1 }\end{array}$ & 28.74 & $\begin{array}{l}\text { External } \\
\text { Defect-2 }\end{array}$ \\
\hline & $\left(-45^{\circ}\right)$ & & 25.76 & \\
\hline $0^{\circ}$ & $\left(-90^{\circ}\right)$ & & 24.58 & \\
\hline $0^{\circ}$ & $\left(+90^{\circ}\right)$ & & 28.74 & \\
\hline & $\left(+45^{\circ}\right)$ & & 28.74 & \\
\hline & $\left(0^{\circ}\right)$ & $\begin{array}{l}\text { Internal } \\
\text { Defect }\end{array}$ & 28.74 & $\begin{array}{l}\text { Internal } \\
\text { Defect-2 }\end{array}$ \\
\hline & $\left(-45^{\circ}\right)$ & & 25.76 & \\
\hline & $\left(-90^{\circ}\right)$ & & 24.34 & \\
\hline & $\left(+90^{\circ}\right)$ & & 28.74 & \\
\hline & $\left(+45^{\circ}\right)$ & & 28.74 & \\
\hline & $\left(0^{\circ}\right)$ & $\begin{array}{l}\text { External } \\
\text { Defot }\end{array}$ & 28.74 & $\begin{array}{l}\text { External } \\
\text { Dect }\end{array}$ \\
\hline & $\left(-45^{\circ}\right)$ & & 26.46 & \\
\hline $225^{\circ}$ & $\left(-90^{\circ}\right)$ & & 24.77 & \\
\hline $22.5^{\circ}$ & $\left(+90^{\circ}\right)$ & & 28.74 & \\
\hline & $\left(+45^{\circ}\right)$ & & 28.74 & \\
\hline & $\left(0^{\circ}\right)$ & $\begin{array}{l}\text { Internal } \\
\text { Defect }\end{array}$ & 28.74 & $\begin{array}{l}\text { Internal } \\
\text { Pect }\end{array}$ \\
\hline & $\left(-45^{\circ}\right)$ & & 26.32 & \\
\hline & $\left(-90^{\circ}\right)$ & & 24.62 & \\
\hline & $\left(+90^{\circ}\right)$ & & 28.74 & \\
\hline & $\left(+45^{\circ}\right)$ & & 28.74 & \\
\hline & $\left(0^{\circ}\right)$ & $\begin{array}{l}\text { External } \\
\text { Defect-1 }\end{array}$ & 28.74 & $\begin{array}{l}\text { External } \\
\text { Defect-2 }\end{array}$ \\
\hline & $\left(-45^{\circ}\right)$ & & 26.88 & \\
\hline $45^{\circ}$ & $\left(-90^{\circ}\right)$ & & 24.77 & \\
\hline $45^{\circ}$ & $\left(+90^{\circ}\right)$ & & 28.74 & \\
\hline & $\left(+45^{\circ}\right)$ & & 28.74 & \\
\hline & $\left(0^{\circ}\right)$ & $\begin{array}{l}\text { Internal } \\
\text { Defect-0 }\end{array}$ & 28.74 & $\begin{array}{l}\text { Internal } \\
\text { Defect-2 }\end{array}$ \\
\hline & $\left(-45^{\circ}\right)$ & & 26.60 & \\
\hline & $\left(-90^{\circ}\right)$ & & 25.16 & \\
\hline & & & M o & el-07 \\
\hline$\alpha$ & $\beta$ & Defect & $\begin{array}{l}\text { FEM } \\
\text { (MPa) }\end{array}$ & Defect \\
\hline & $\left(+90^{\circ}\right)$ & & 17.24 & \\
\hline & $\left(+45^{\circ}\right)$ & & 17.24 & \\
\hline & $\left(0^{\circ}\right)$ & External & 17.06 & External \\
\hline & $\left(-45^{\circ}\right)$ & & 15.80 & \\
\hline & $\left(-90^{\circ}\right)$ & & 14.41 & \\
\hline $0^{\circ}$ & $\left(+90^{\circ}\right)$ & & 17.24 & \\
\hline & $\left(+45^{\circ}\right)$ & & 17.24 & \\
\hline & $\left(0^{\circ}\right)$ & Internal & 17.10 & Internal \\
\hline & $\left(-45^{\circ}\right)$ & & 15.63 & \\
\hline & $\left(-90^{\circ}\right)$ & & 14.33 & \\
\hline & $\left(+90^{\circ}\right)$ & & 17.24 & \\
\hline & $\left(+45^{\circ}\right)$ & & 17.24 & \\
\hline & $\left(0^{\circ}\right)$ & $\begin{array}{l}\text { External } \\
\text { Defotat }\end{array}$ & 17.24 & External \\
\hline & $\left(-45^{\circ}\right)$ & & $\begin{array}{l}15.88 \\
\end{array}$ & \\
\hline $225^{\circ}$ & $\left(-90^{\circ}\right)$ & & 14.58 & \\
\hline $22.5^{\circ}$ & $\left(+90^{\circ}\right)$ & & 17.24 & \\
\hline & $\left(+45^{\circ}\right)$ & & 17.24 & \\
\hline & $\left(0^{\circ}\right)$ & Internal & 17.15 & Internal \\
\hline & $\left(-45^{\circ}\right)$ & & 15.80 & \\
\hline & $\left(-90^{\circ}\right)$ & & $\begin{array}{l}14.66 \\
\end{array}$ & \\
\hline
\end{tabular}




\begin{tabular}{|c|c|c|c|c|c|c|c|c|c|c|c|c|c|c|c|}
\hline \multirow{10}{*}{. } & $\left(+90^{\circ}\right)$ & \multirow{4}{*}{$\begin{array}{l}\text { External } \\
\text { Defect-1 }\end{array}$} & 17.24 & \multirow{4}{*}{$\begin{array}{l}\text { External } \\
\text { Defect-2 }\end{array}$} & 17.24 & \multirow{4}{*}{$\begin{array}{l}\text { External } \\
\text { Defect-3 }\end{array}$} & 10.40 & \multirow{10}{*}{$45^{\circ}$} & \multirow{4}{*}{\begin{tabular}{|c|}
$\left(+90^{\circ}\right)$ \\
$\left(+45^{\circ}\right)$ \\
$\left(0^{\circ}\right)$ \\
$\left(-45^{\circ}\right)$ \\
\end{tabular}} & \multirow{5}{*}{$\begin{array}{l}\text { External } \\
\text { Defect-1 }\end{array}$} & \multirow{3}{*}{$\begin{array}{l}13.77 \\
13.77 \\
13.77 \\
\end{array}$} & \multirow{5}{*}{$\begin{array}{l}\text { External } \\
\text { Defect-2 }\end{array}$} & \multirow{3}{*}{$\begin{array}{l}13.77 \\
13.72 \\
12.67 \\
\end{array}$} & \multirow{5}{*}{$\begin{array}{l}\text { External } \\
\text { Defect-3 }\end{array}$} & \multirow{3}{*}{$\begin{array}{l}8.40 \\
8.15 \\
7.28\end{array}$} \\
\hline & $\left(+45^{\circ}\right)$ & & 17.24 & & 17.24 & & 10.05 & & & & & & & & \\
\hline & & & 17.24 & & 15.62 & & 9.70 & & & & & & & & \\
\hline & $\left(-45^{\circ}\right)$ & & 15.96 & & 13.92 & & 8.74 & & & & 12.86 & & 11.24 & & 6.76 \\
\hline & $\left(-90^{\circ}\right)$ & & 14.69 & & 12.83 & & 8.23 & & $\left(-90^{\circ}\right)$ & & 11.72 & & 10.40 & & 6.36 \\
\hline & $\left(+90^{\circ}\right)$ & & 17.24 & & 17.33 & & 10.45 & & $\left(+90^{\circ}\right)$ & & 13.77 & & 13.77 & & 8.31 \\
\hline & $\left(+45^{\circ}\right)$ & & 17.24 & & 17.13 & & 10.00 & & $\left(+45^{\circ}\right)$ & & 13.77 & & 13.77 & & 8.19 \\
\hline & $\left(0^{\circ}\right)$ & $\begin{array}{l}\text { Internal } \\
\text { Defect-01 }\end{array}$ & 17.24 & $\begin{array}{l}\text { Internal } \\
\text { Defect-2 }\end{array}$ & 15.70 & $\begin{array}{l}\text { Internal } \\
\text { Defect-3 }\end{array}$ & 9.64 & & $\left(0^{\circ}\right)$ & $\begin{array}{l}\text { Internal } \\
\text { Defect-0 }\end{array}$ & 13.77 & $\begin{array}{l}\text { Internal } \\
\text { Defect-2 }\end{array}$ & 12.81 & $\begin{array}{l}\text { Internal } \\
\text { Defect-3 }\end{array}$ & 7.34 \\
\hline & $\left(-45^{\circ}\right)$ & Defect-01 & 15.96 & Defect-2 & 13.86 & Defect-3 & 8.62 & & $\left(-45^{\circ}\right)$ & Defect-01 & 13.00 & Defect-2 & 11.29 & Defect-3 & 6.64 \\
\hline & $\left(-90^{\circ}\right)$ & & 14.90 & & 12.90 & & 8.15 & & $\left(-90^{\circ}\right)$ & & 11.53 & & 10.32 & & 6.31 \\
\hline
\end{tabular}

Table 5 shows the finite element simulation results for elbows with all positions and sizes of defect: three sizes, three angles of $\beta$ and five angles of $\alpha$.

\section{Results and Discussion}

\subsection{Fitting and comparison of limit loads}

This assessment of damaged elbows goes beyond the stress distribution characteristics of elbows covered by the Goodall formula. Fig. 8 shows the stress distribution characteristics of elbows based on the FE analysis results. The limit load calculation of an elbow without defect is simple, and structural failure occurs on the intrados as a result of the stress distribution. However, defects or mass losses change the stress distribution of elbows asymmetrically. As seen in Fig. 8, the structural failure position changes with defect position at the mean angle $\alpha$ and at the bending angle $\beta$. Because of the symmetric modelling of the elbow, the range of angle is defined $90^{\circ} \sim-90^{\circ}$ for $\alpha$ and $0^{\circ} \sim 45^{\circ}$ for $\beta$. This range covers all positions of the mean and bending angles.

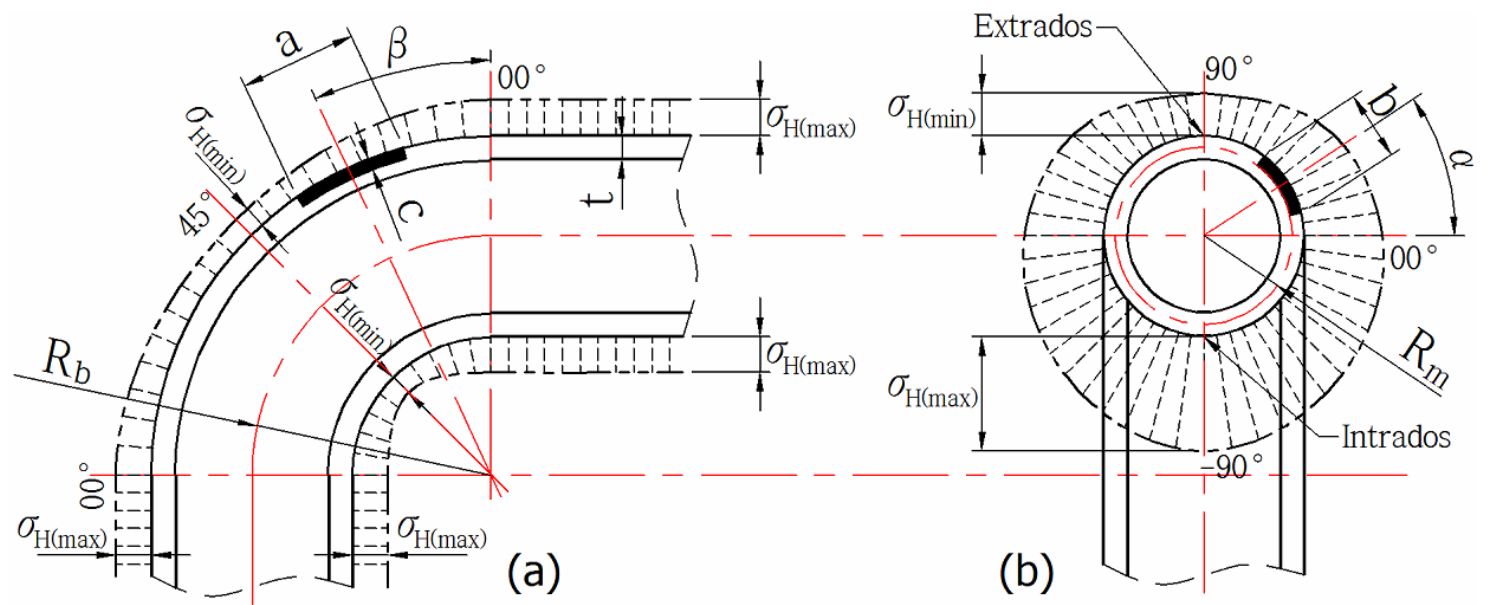

Fig.7. (a) Stress distribution of the elbow at the bending angle. (b) Stress distribution of the elbow at the mean angle

The bending factor $(B F)$ is an indicator of the increase or decrease in the nominal stresses in the bending radius of an elbow relative to a straight pipe, as shown in Fig. 7(a). The mean factor $(M F)$ is an indicator of the increase or decrease in the nominal stresses in the mean radius of an elbow relative to a straight pipe, as shown in Fig. 7(b). These factors were found by fitting the FE simulation results of several elbows with different defect positions. The factors $B F$ and $M F$ are defined as follows: 


$$
\begin{aligned}
& B F=1+\sin \left(\frac{\beta}{24}\right) \\
& M F=\left[\frac{\frac{R_{b}}{R_{m}}+\sin \alpha}{\frac{R_{b}}{R_{m}}+\frac{\sin \alpha}{1.502}}\right]
\end{aligned}
$$

Defect size is another factor that changes the stress distribution and maximum limit load of elbows with defects. Three sizes of defect with several angles of mean and bending radius were defined for the FEA. The simplified assumed defect is shown in Fig. 2. and the size of the applied defects is shown in Table 2. These defects were applied to all sizes of elbow internally and externally. A factor $D F$ for elbows with defects was derived by FEA results data fitting as:

$$
D F=\left(1-\left[\left(\frac{a}{2 \pi R_{b}}\right)^{1.5}+\left(\frac{b}{2 \pi R_{m}}\right)^{1.5}+\left(\frac{c}{t}\right)^{3.2}\right]\right)
$$

As mentioned, Goodall proposed the formula for the limit pressure $P_{f}$. However, it only calculates the limit load of elbows without defects. Duan and Shin (2006) suggested a modified formula that covers elbows with certain positions of defect (centre position of bending angle on extrados). In the modified Goodall formula only the size of the defect affects the limit load of elbows, with no consideration given to position. As a result, a new formula for the maximum limit load of elbows that covers elbows both with and without defects in all positions was developed. This formula has two parts to reflect the stress distributions along the mean angle and bending angle.

$$
\text { if } \mid \begin{aligned}
& a \leq \sqrt{100 R_{m} t} \\
& c<0.5 t \\
& \alpha \geq 0^{\circ}
\end{aligned} \Rightarrow P_{f d}=\frac{\sigma_{f} t}{R_{m}} \frac{1-R_{m} / R_{b}}{1-R_{m} /\left(2 R_{b}\right)}
$$

Otherwise

$$
P_{f d}=\frac{\sigma_{f} t}{R_{m}} \frac{1-R_{m} / R_{b}}{1-R_{m} /\left(2 R_{b}\right)} \times D F \times M F \times B F
$$

Where

$$
D F=\left(1-\left[\left(\frac{a}{2 \pi R_{b}}\right)^{1.5}+\left(\frac{b}{2 \pi R_{m}}\right)^{1.5}+\left(\frac{c}{t}\right)^{3.2}\right]\right)
$$




$$
\begin{aligned}
& M F=\left[\frac{\frac{R_{b}}{R_{m}}+\sin \alpha}{\frac{R_{b}}{R_{m}}+\frac{\sin \alpha}{1.502}}\right] \\
& B F=1+\sin \left(\frac{\beta}{24}\right)
\end{aligned}
$$

The formula shows that if the defect is defined as short (Modified ASME B $31 \mathrm{G}, a \leq \sqrt{100 R_{m} t}$ ), the depth of the defect is less than half the elbow wall thickness and the defect is located on the extrados $\left(\alpha \geq 0^{\circ}\right)$, then the stress distribution of the elbow does not change and structural failure appears on the intrados. The defect efficiency is ignored for case (5). For other positions and sizes of defect, formula (6) is valid.

All of the significant behavioural effects that influence the thermal and geometric characteristics of elbow pipeline for operating, hydraulic and service fluids should be described. It is important to realise that the modelling technique applied must be capable of representing the actual structural behaviour associated with geometrical nonlinearity, material nonlinearity (temperature), type and magnitude of initial imperfections (ovality) due to installation and transportation, boundary conditions, loading conditions and mesh size, etc. However, in this paper is only considered defect effect firstly. It should be clarified further study.

\subsection{Comparison of the FEA results with the developed formula}

The limit load of all elbows with several sizes of defect and position was calculated by FEA and then compared with limit loads calculated with the new formula. Fig. 8 shows the results from the FEA results (blue curves) and the new formula results (red curves).
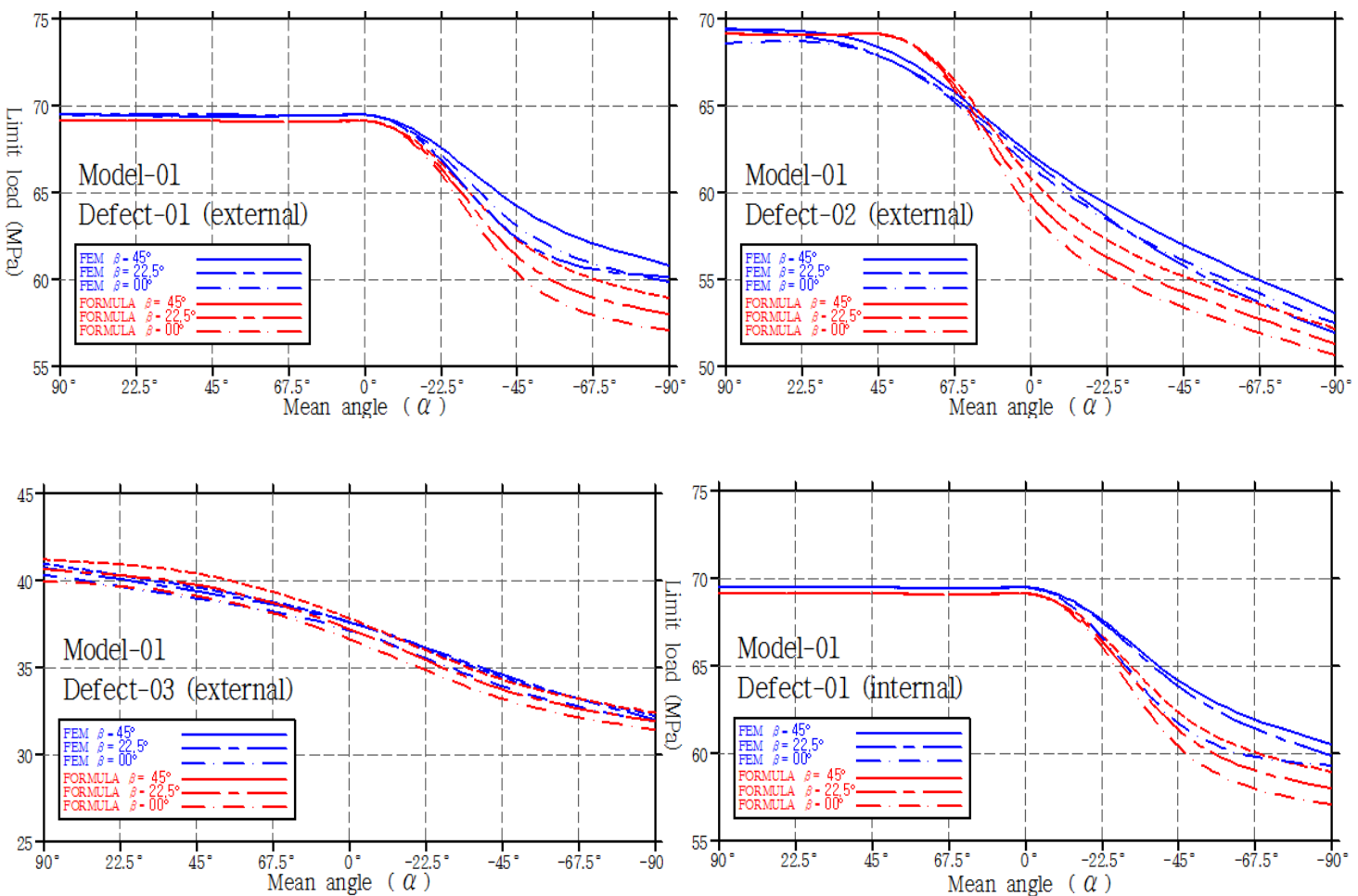

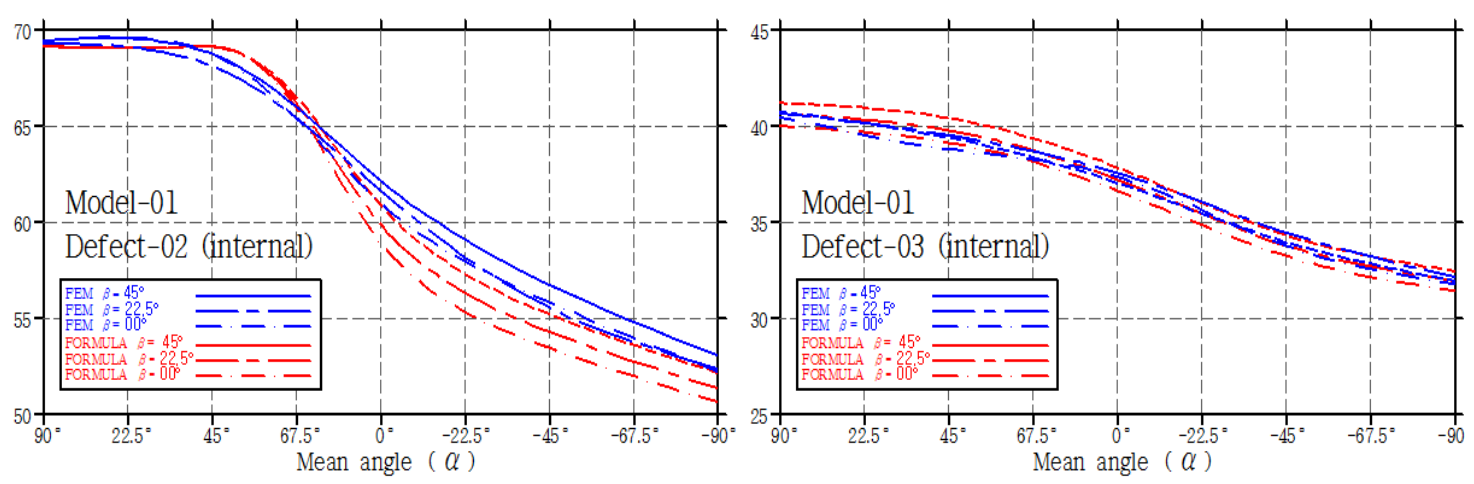

Fig. 8. Comparison of the limit loads of elbows with defects calculated by the FEA and the new formula (continued in Appendix )

Fig. 8 shows that the limit loads of elbows with defects calculated by the new formula are comparable to those calculated by FEA for all of the selected models with various sizes and positions of defect.

\section{Conclusion}

The following conclusions are drawn from the calculation of the maximum limit loads of elbows with and without defects by FEA and the new formula.

1. The new formula results are consistent with those of the FEA, indicating that the modelling method is valid. The formula is effective for calculating the limit load of elbows with defects under internal pressure.

2. FEA shows that the limit loads of all elbows with defects significantly change with the mean angle, bending angle and size of defect. However, if the defect is located on the extrados and the depth of the defect is less than half the material thickness, elbows with defects burst in a standard manner and structural failure appears on the intrados. In this case, the limit state of failure of an elbow with a defect is identical to the corresponding elbow without a defect.

3. An empirical formula of the limit load for elbows with defects in all positions on the bending part of the elbow has been proposed by data fitting the FEA results to calculate the maximum limit load of elbows with defects more accurately than the existing formula, which is conservative.

These conclusions are applicable for several sizes of elbow with similar stress-strain curves. However, the shapes of the plastic stage and the stress and strain values need not be the same as those in this study, because the formula includes the flow stress of the material. To obtain more general conclusions, it is suggested that future studies take different material properties into consideration to attain a variety of results for the maximum limit load pressure of elbows with defects.

\section{Acknowledgements}

The study was undertaken at the Lloyd's Register Foundation Research Centre of Excellence at Pusan 
National University. Lloyd's Register Foundation (LRF), a UK registered charity and sole shareholder of Lloyd's Register Group Ltd, invests in science, engineering and technology for public benefit, worldwide.

\section{References}

An JH, Hong SP, Kim YJ, Budden PJ. 2011. Elastic stresses for 90 degrees elbows under in-plane bending. International Journal of Mechanical Sciences 53: 762-776

ANSYS. 2012. ANSYS User Manual (Release 14.0). PA: ANSYS Inc.

ASME BPVC. 2010. Rules for construction of pressure vessels. Boiler and Pressure Vessel Code Section VIII division 2. New York: American Petroleum Institute.

Calladine CR. 1974. Limit analysis of curved tubes. Journal of Mechanical Engineering Science 16: 85-87, 1974.

Chattopadhyay J. 2002. The effect of internal pressure on in-plane collapse moment of elbows. Nuclear Engineering and Design 212: 133-144

Duan ZX, Shen SM. 2006. Analysis and experiments on the plastic limit pressure of elbows. International Journal of Pressure Vessels and Piping 83: 707-713.

Firoozabad ES, Jeon BG, Choi HS, Kim NS. 2015. Seismic fragility analysis of seismically isolated nuclear power plantspiping system. Nuclear Engineering and Design 284: 264-279.

Goodall IW. 1978. Lower bound limit analysis of curved tubes loaded by combined internal pressure and in-plane bending moment. RD/B/ N4360, CEGB; 1978.

Guo C. 1999. Plastic limit loads for surface defect pipes and bends under combined loads of tension, bending, torsion and internal pressure. East China University of Science and Technology.

Han JJ, Lee KH, Kim NH, Kim YJ, Jeong DW, Budden PJ. 2012. Comparison of existing plastic collapse load solutions with experimental data for 90degrees elbows. International Journal of Pressure Vessels and Piping 89: 19-27

Han LH, Liu ZD. 1998. Limit load analysis for local wall thinning pipeline under internal pressure. Press Ves Technology 15(4): 1-4.

Kim JW, Lee SH, Park CY. 2009. Experimental evaluation of the effect of local wall thinning on the failure pressure of elbows. Nuclear Engineering and Design 239: 2737-2746.

Kim YJ, Kim JH, An JH, Hong SP, Park CY. 2008. Effects of local wall thinning on plastic limit loads of elbows using geometrically linear FE limit analyses. Engineering Fracture Mechanics 75: 2225-2245

Lee KH, Oh CS, Kim YJ, Yoon KB. 2009. Quantification of the yield strength-to-elastic modulus ratio 
effect on TES plastic loads from finite element limit analyses of elbows. Engineering Fracture Mechanics 76: 856-875.

Oh CS, Kim YJ, Park CY. 2008. Shakedown limit loads for elbows under internal pressure and cyclic inplane bending. International Journal of Pres Vessels and Piping.85:394-405

Srivastava A, Prabhakaran KM, Ghosh AK. 2011. Studies on the behavior of part-through circumferential crack at intrados in elbows under in-plane bending moment. Nuclear Engineering and Design 241: 2386-2397.

Wang Y, Liu S, Fan DS. 2005. Plastic limit load analysis of elbow with local thinning. J Beijing Univ Chem Technology 32(1): 62-65.

Zhang L, Wang Y, Liu CJ, Liu C. 2001. Evaluation of local thinned pressurized elbows. International Journal of Pressure Vessels and Piping 78: 697-703.

Appendix. Comparison of the limit loads of elbows with defects calculated by the FEA and the new formula (Fig. 8 is contined ) 

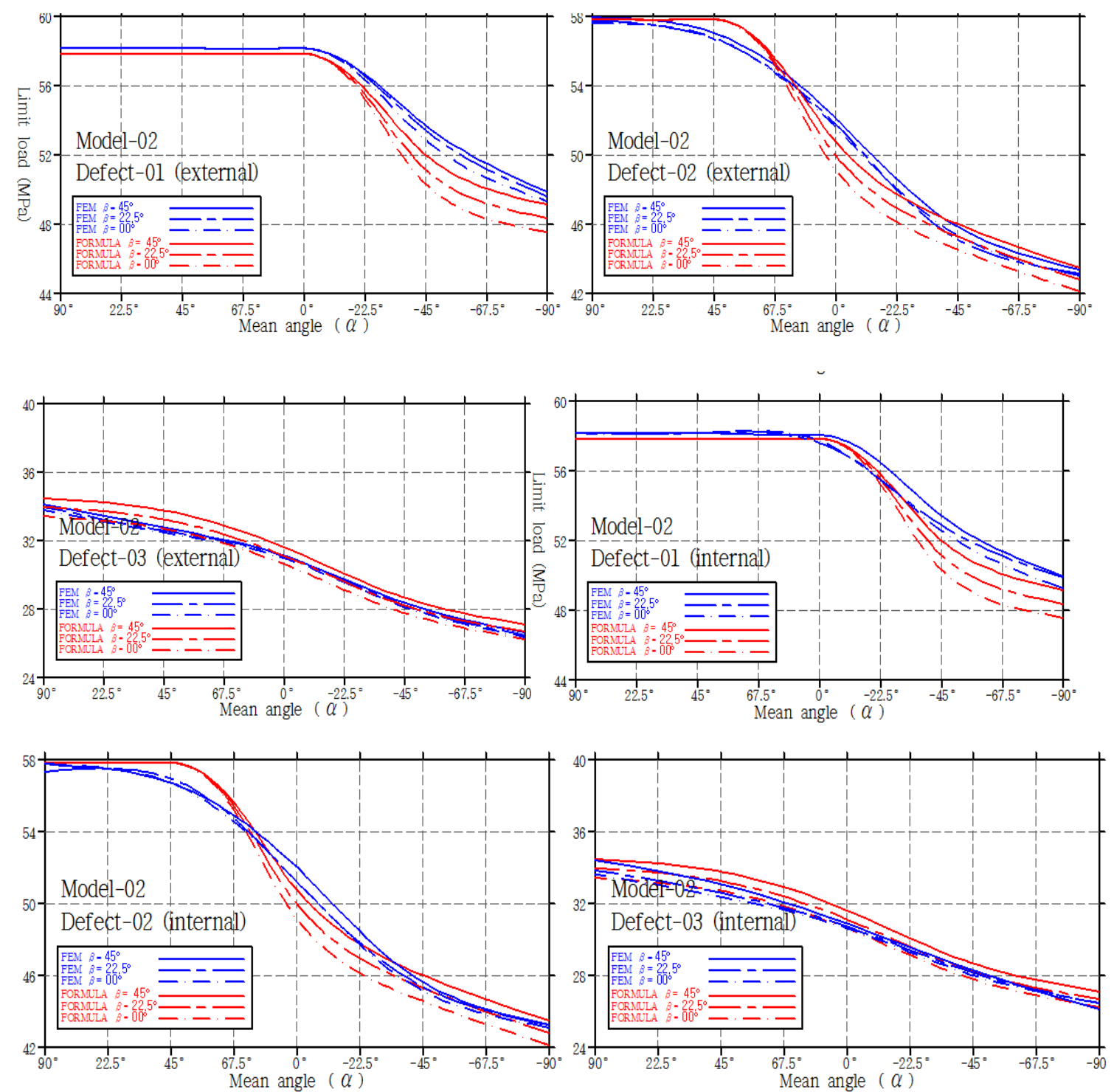

(a) Model -02 with varying defects
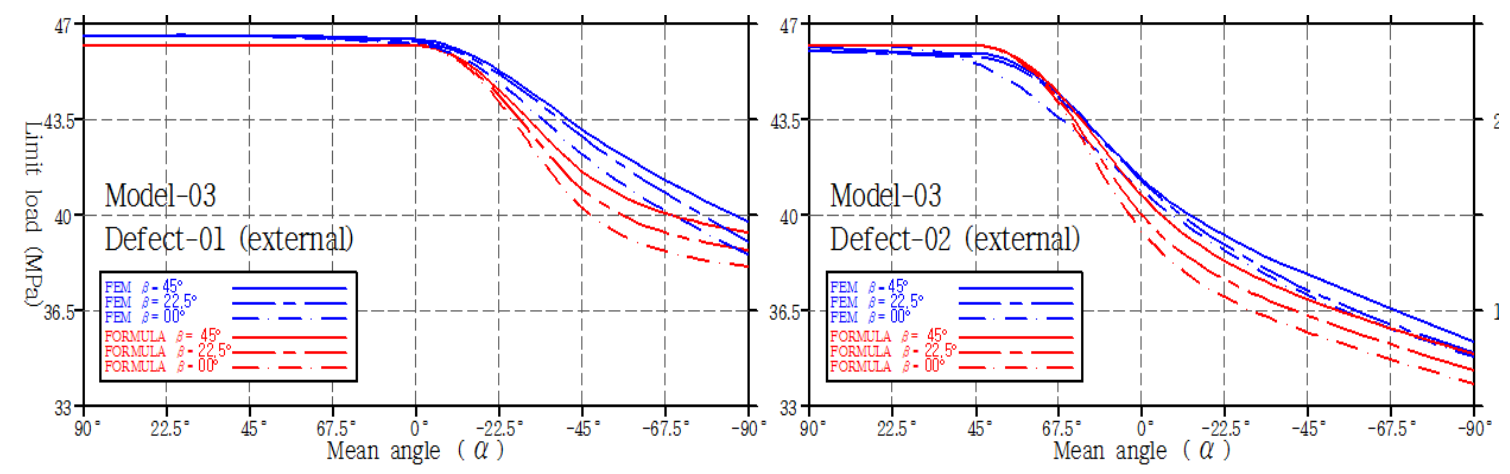

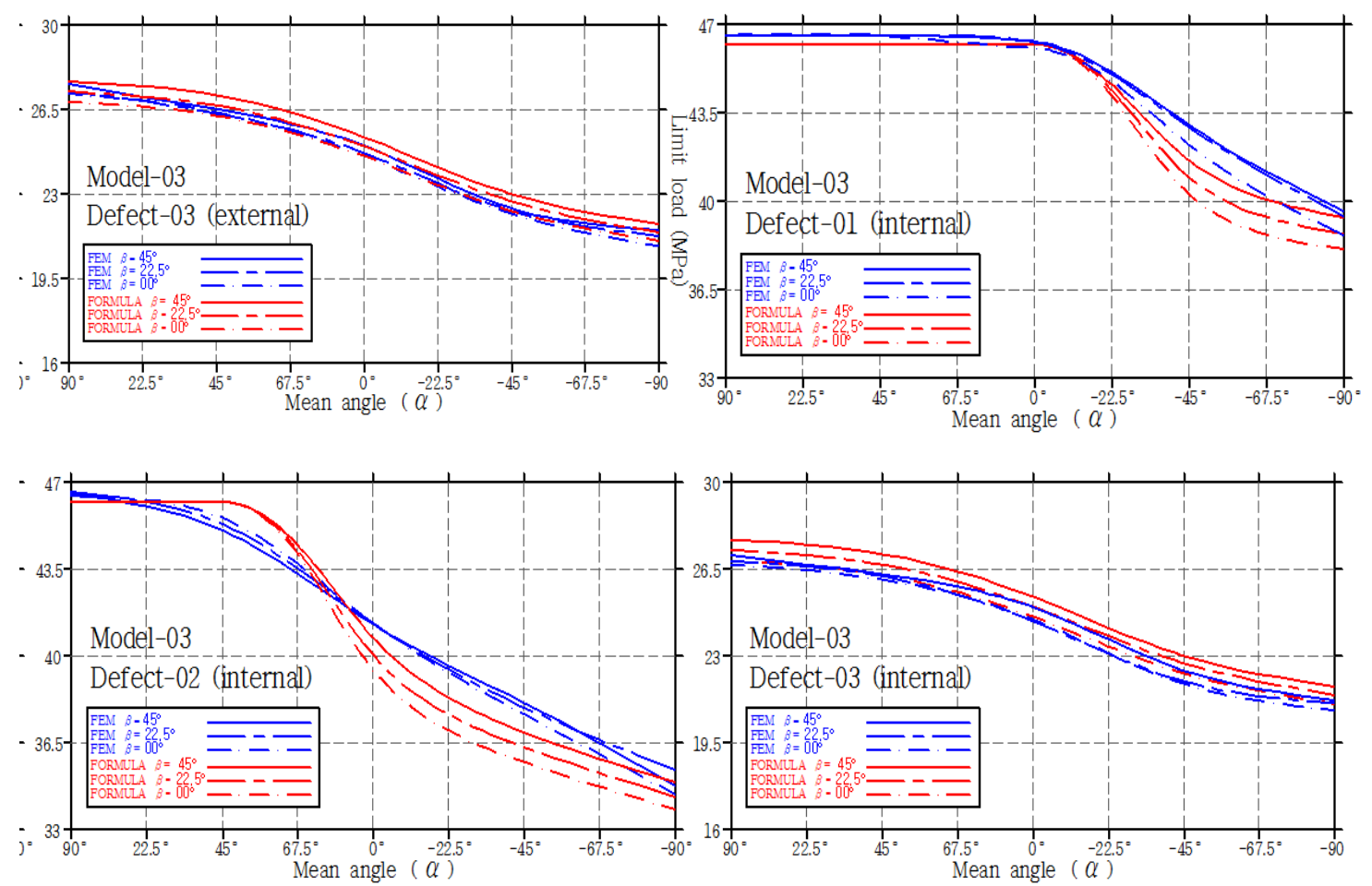

(b) Model -03 with varying defects
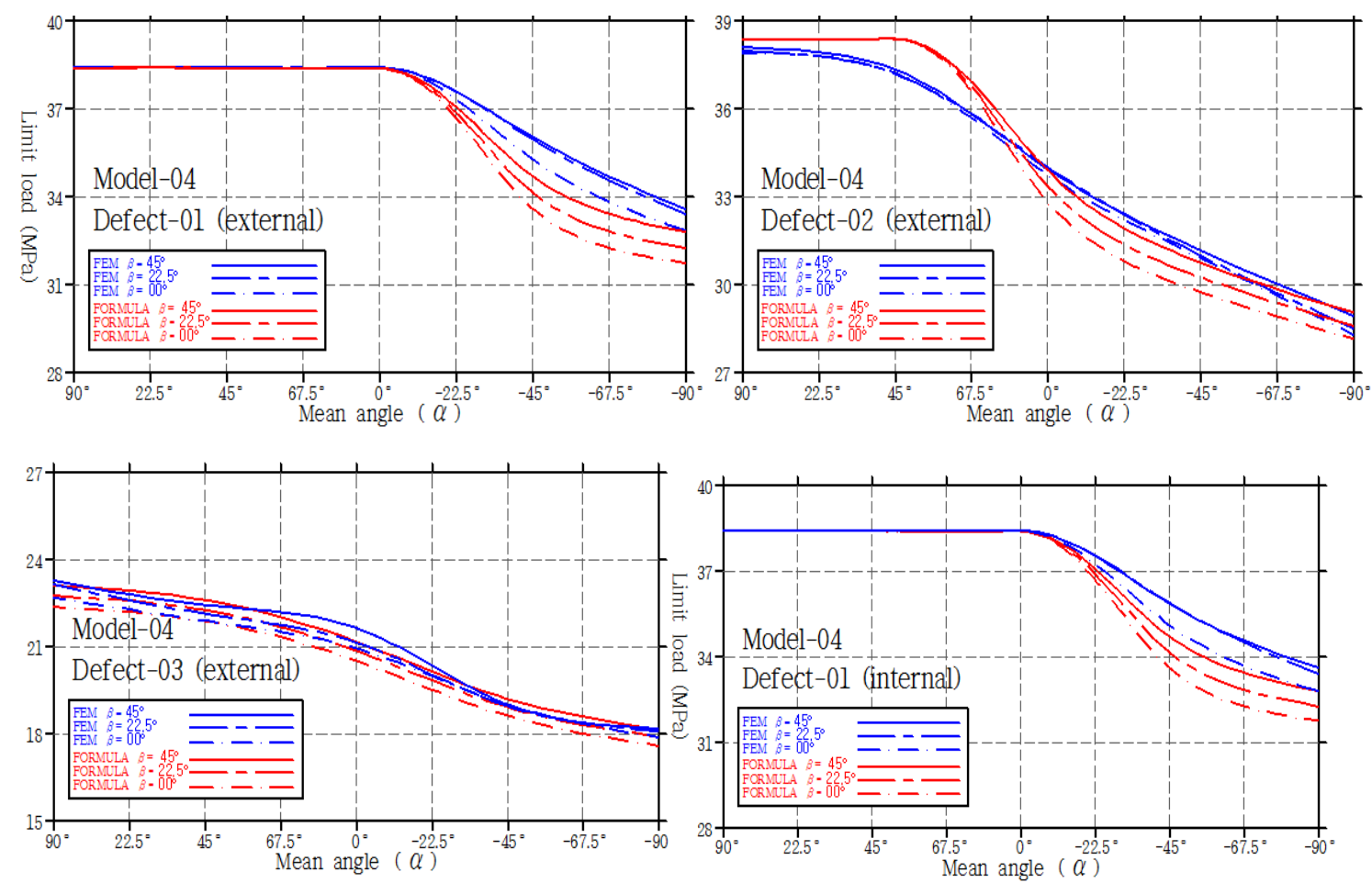

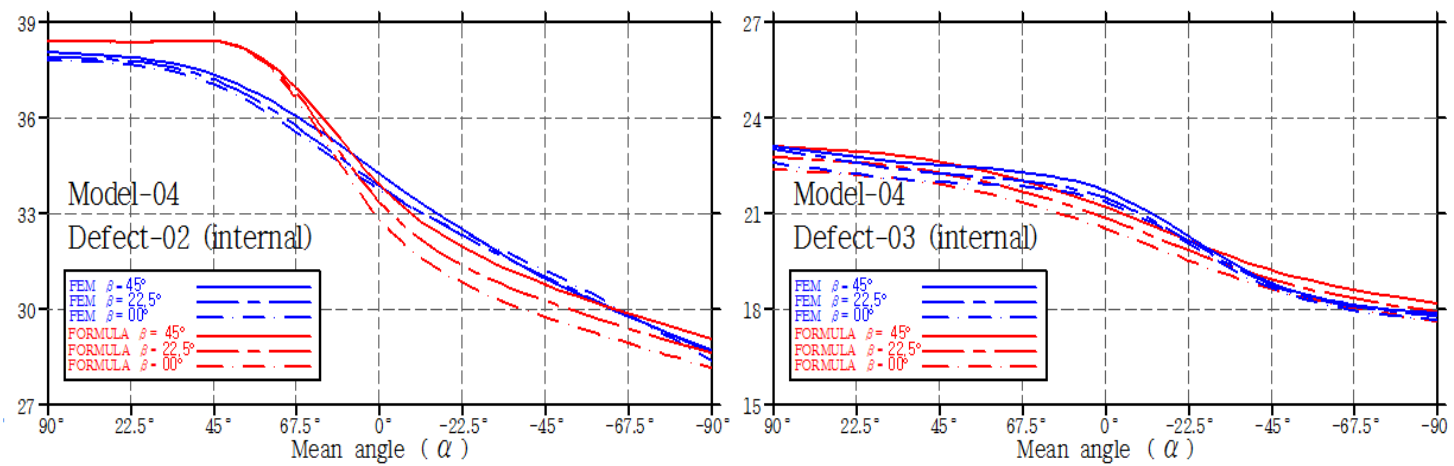

(c) Model -04 with varying defects
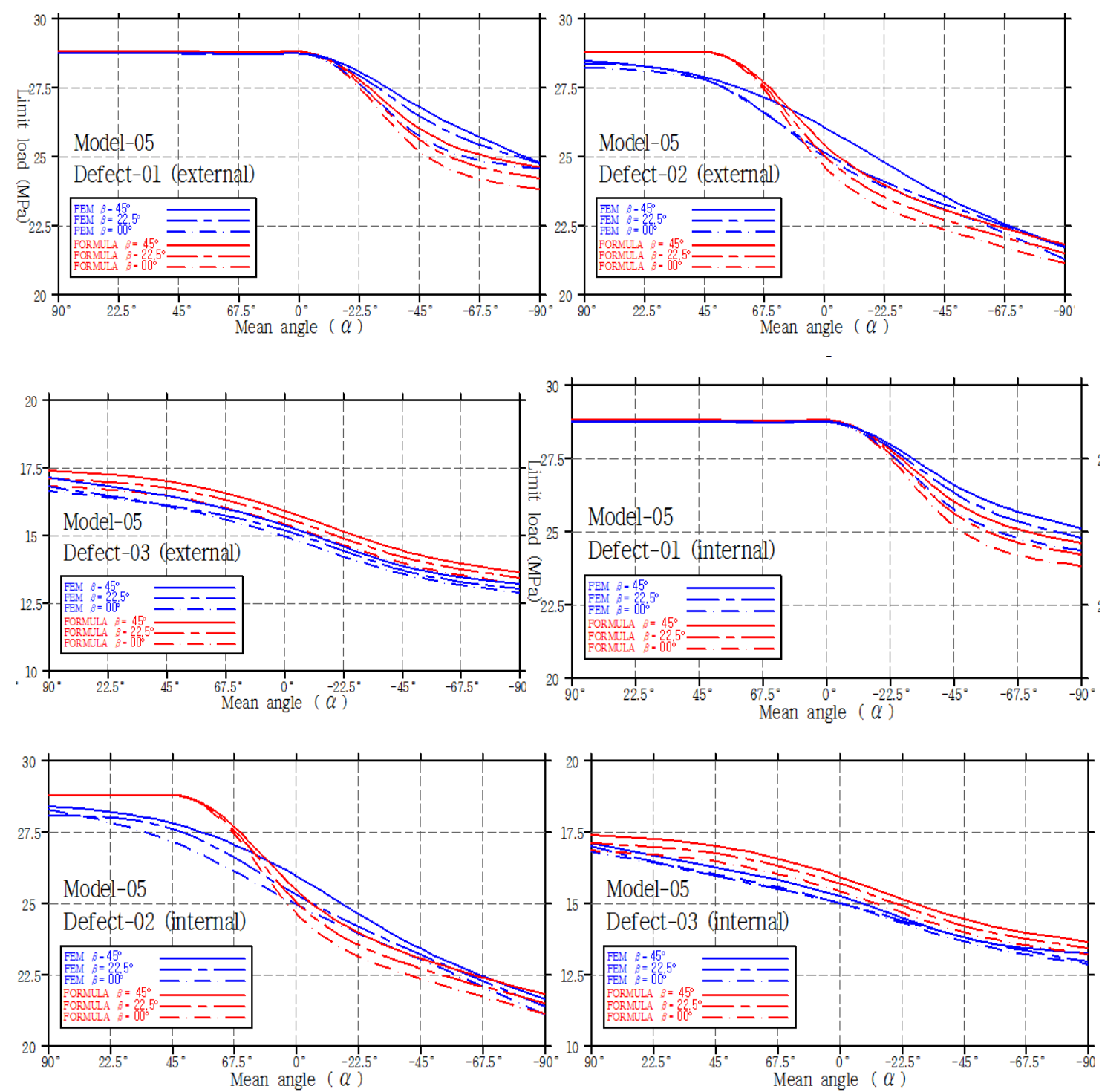

(d) Model -05 with varying defects 

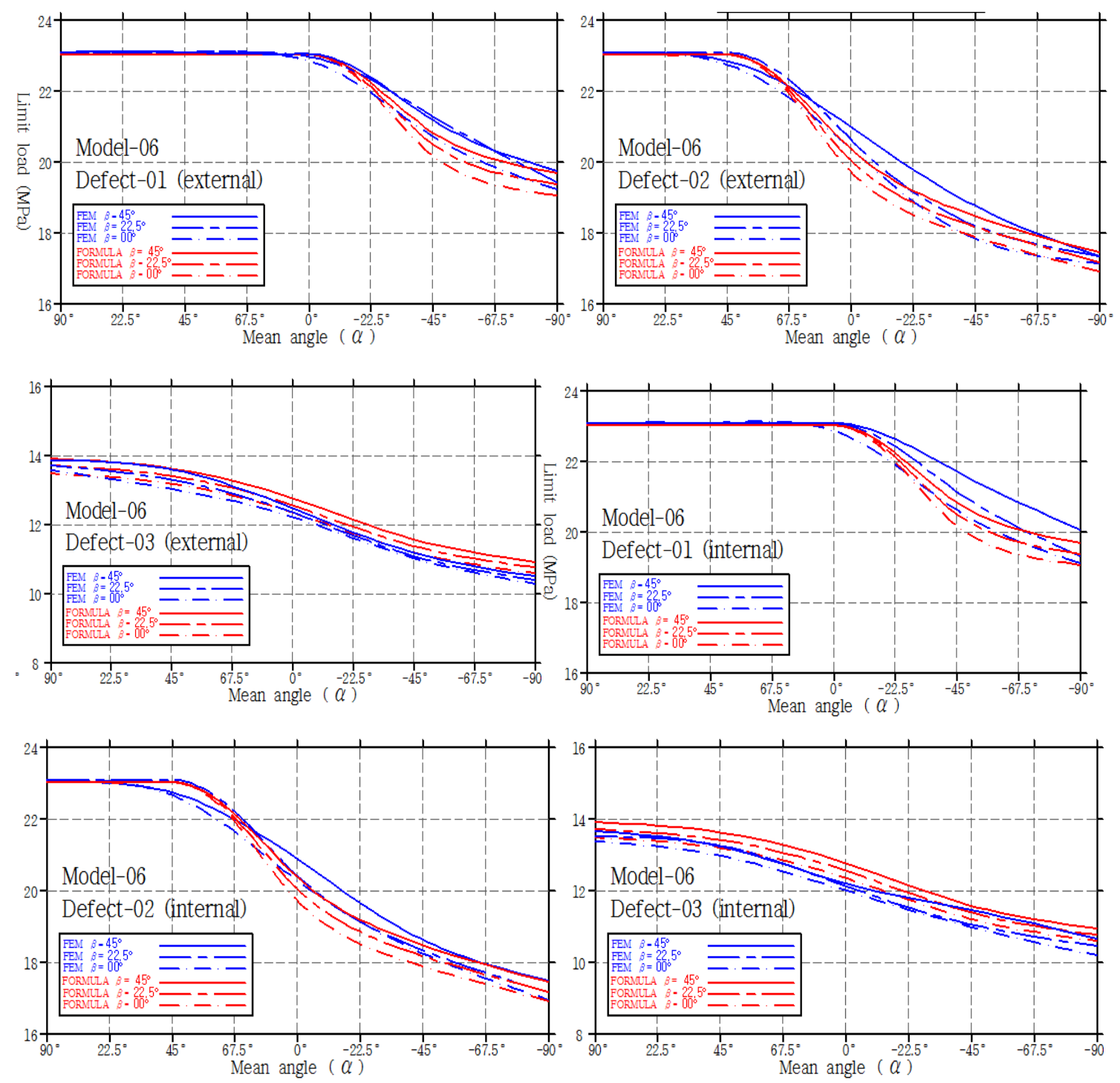

(e) Model -06 with varying defects
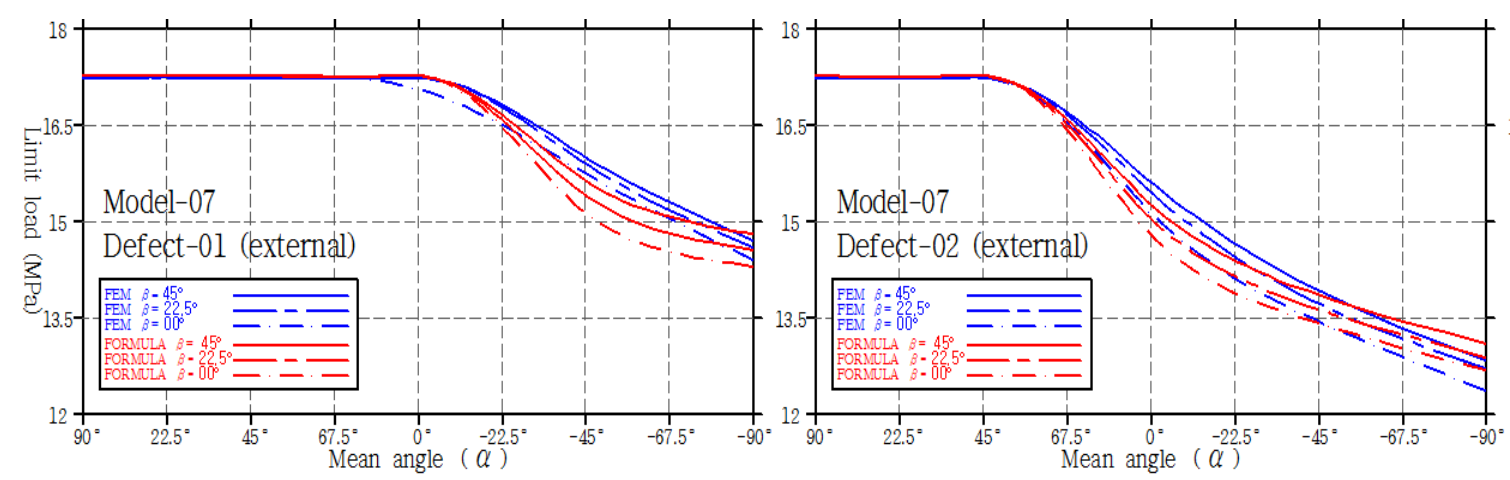

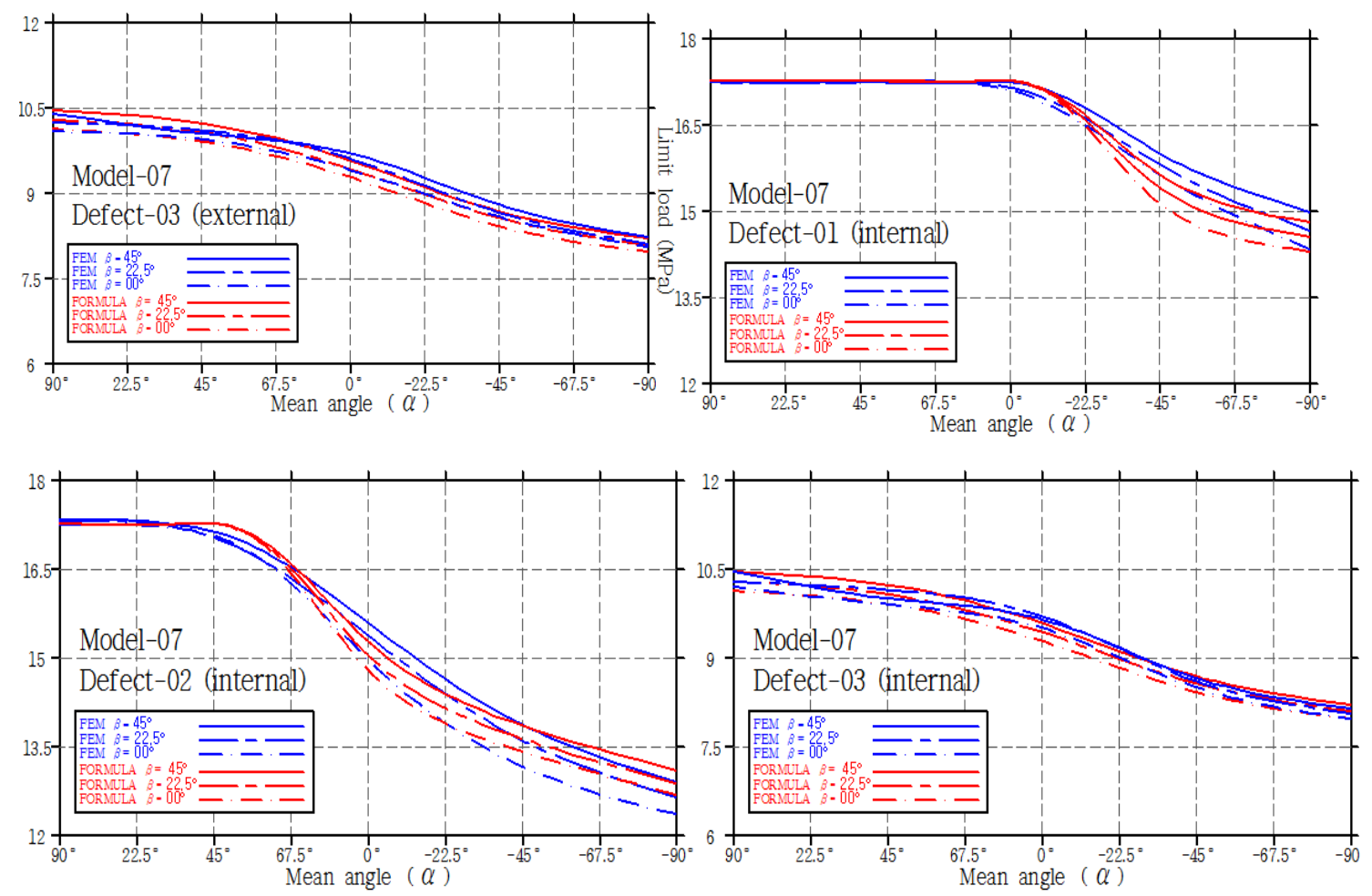

(f) Model -07 with varying defects
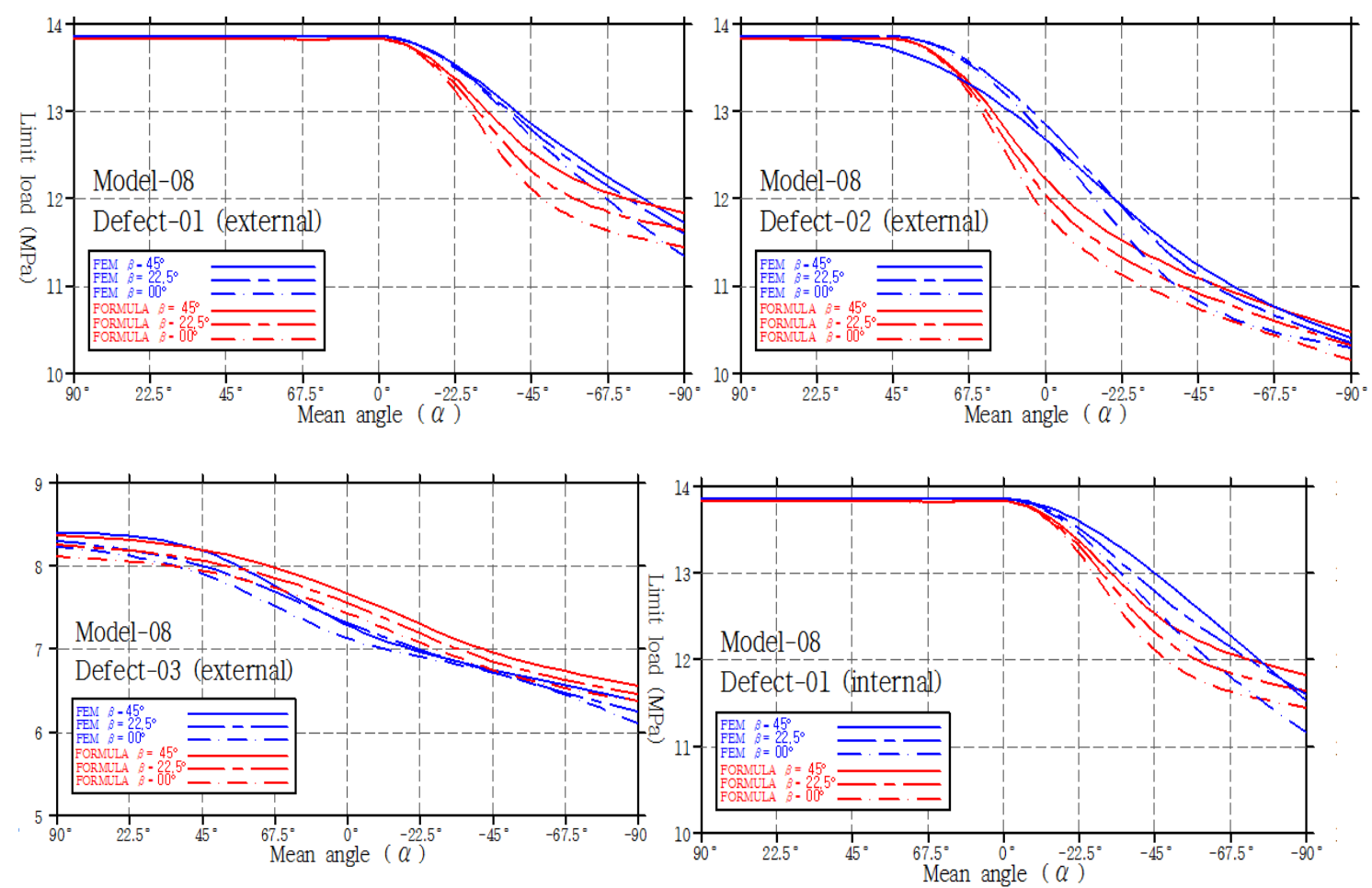

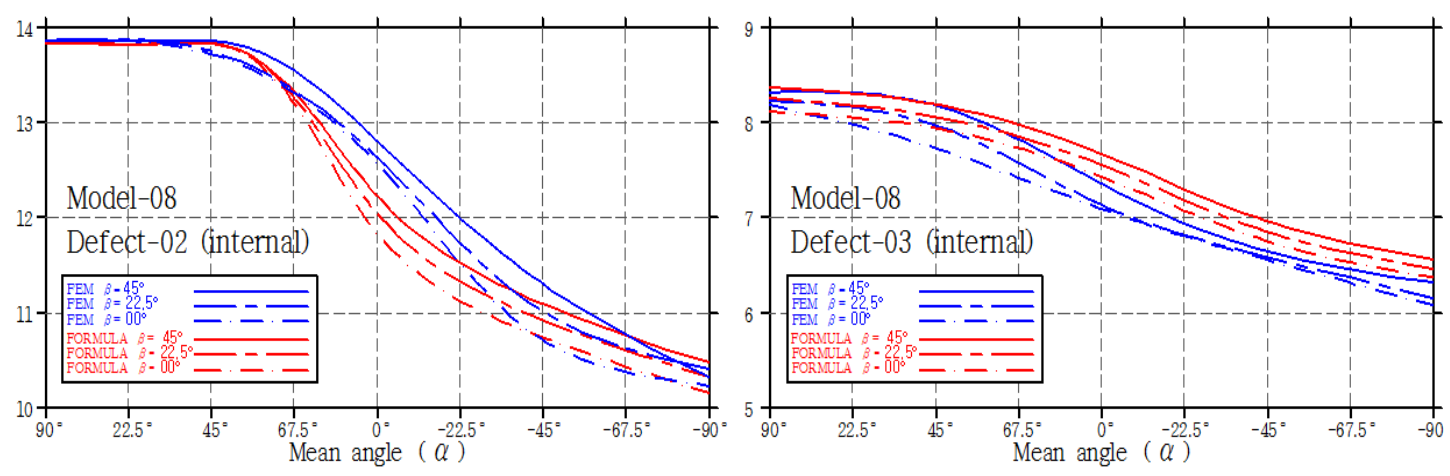

(g) Model -07 with varying defects 